\title{
IMPROVED SURFACE FLATNESS OF A Bi-SUBSTITUTED DY IRON GARNET FILM FOR MAGNETO-OPTICAL RECORDING BY SPUTTER-ETCHING
}

\author{
R. SATO, N. KAWAMURA and T. TAMAKI
}

\begin{abstract}
Advanced Materials \& Devices Research Division, NHK Science \& Technical Research Laboratories 1-10-11 Kinuta, Setagaya-ku, Tokyo 157, Japan
\end{abstract}

\begin{abstract}
Bi which precipitates near the surface of as-deposited film is the origin of surface roughness of Bi-substituted garnet film. The Bi-rich thin layer can be effectively removed from the film surface by successively sputter-etching a film after deposition for a period equal to $1 / 12$ of sputtering time. The averaged surface roughness of garnet film crystallized from this state is improved by up to $1 / 4$ compared that of the non-etched sample. Regular shaped bits can be recorded on the garnet bi-layered medium each layer of which was processed using this method. KEYWORDS : GARNET , MAGNETO-OPTICAL , BISMUTH , PRECIPITATION, SPUTTER-ETCHING , FLATNESS
\end{abstract}

\section{INTRODUCTION}

A Bi- and Ga-substituted Dy iron garnet(Bi,Ga:DyIG) film deposited on a glass substrate and crystallized by post annealing becomes a poly-crystalline film. As a result, for magneto-optical recording applications there exist some undesirable properties, such as grain boundary, crack, surface roughness, and inhomogeneity of compositional ratio.

The authors have reported before that a flat Bi,Ga:DyIG single phase film with an averaged glass substrate using
surface roughness of $2 \mathrm{~nm}$ can be RF-sputtering method. prepared on a glass substrate with a thermal expansion ratio of $98 \times 10^{-7} /{ }^{\circ} \mathrm{C}$ by mixing 5 at. $\%$ of oxygen with Ar[1]. But, unfortunately, the shape of the Faraday hysteresis loop(FL) of the garnet films prepared under these conditions was not square.

Furthermore, FL's having complex shapes which reflect dispersion in compensation temperature were often observed. These results imply that there still remains compositional inhomogeneity which may reduce recording properties of a garnet film.

The purpose of this work is to develop a Bi,Ga:DyIG film without the inhomogeneity mentioned above. Firstly, we describe in detail the compositional inhomogeneity which exists in a sample after and/or before crystallization. Secondly, we introduce methods and preparation conditions to improve homogeneity. Finally, bit recording data in a $\mathrm{Bi}, \mathrm{Ga}$ :DyIG and $\mathrm{Bi}$ - and Ga-substituted yttrium iron garnet(Bi,Ga:YIG) bi-layered medium will be shown.

\section{EXPERIMENTAL}

A film was deposited on a RF-sputtering method. After

deposition, the film was sputter-etched in the same vacuum chamber. An infrared image furnace was used for annealing. Detailed sputtering conditions and the annealing conditions are listed in Tab.1 and Tab.2, respective$1 y$.

Crystallinity of the annealed sample was characterized by an $X$-ray diffraction(XRD) measurement. The $X$-ray tube target is $\mathrm{Cu}$. Surface morphology was observed by an atomic force microscope(AFM). Depth profiles of constituent elements were measured by auger electron spectroscopy(AES). The shape and 
Tab.1 Sputtering and etching conditions.

\begin{tabular}{|c|c|}
\hline SPUTTERING GAS & Ar: $\mathrm{O}_{2}=\overline{95: 5}$ \\
\hline GAS PRESSURE & $40 \mathrm{mTorr}$ \\
\hline SPUTTERING POWER & $100 \mathrm{~W}$ \\
\hline SPUTTERING TIME & I hours \\
\hline FILM THICKNESS & $120 \mathrm{~nm}$ \\
\hline TARGET COMPOSITION & $\begin{array}{l}\mathrm{Bi}_{3.0^{\mathrm{Dy}}} 0.6^{\mathrm{Ga}_{1} .0^{\mathrm{Fe}} 3.3^{\mathrm{O}} \mathrm{x}} \\
\mathrm{Bi}_{3.0^{\mathrm{Y}_{0}} 6^{\mathrm{Ga}_{1}} .7^{\mathrm{Fe}}} . .6^{\mathrm{O}} \mathrm{x}\end{array}$ \\
\hline SUBSTRATE & CORNING0317 \\
\hline ETCHING TIME & 5 minutes \\
\hline
\end{tabular}

Tab.2 Annealing conditions.

\begin{tabular}{|l|l|}
\hline ATMOSPHERE & AIR \\
\hline HEATING RATE & $50^{\circ} \mathrm{C} / \mathrm{s}$ \\
\hline TEMPERATURE & $600^{\circ} \mathrm{C}$ \\
\hline HOLD TIME & 1 hourS \\
\hline
\end{tabular}

distance of bits recorded on a single-layered Bi,Ga:DyIG film and a Bi,Ga:DyIG/Bi,Ga:YIG bi-layered film were compared.

\section{RESULTS AND DISCUSSION}

We performed XRD measurements to analyze minutely the inhomogeneity in our films. As indicated by an arrow in Fig.1, a rather broad peak which can not be ascribed to garnet was present at 27.7 degrees in the XRD pattern of $0-20$ scan. This peak disappears in the pattern with an incident $X$-ray fixed at 1 degree. This result implies that there are non-garnet phases in deep regions of the film and also an accompanying compositional inhomogeneity. A depth profile of AES in Fig.2(a) shows $\mathrm{Bi}$ density has a peak at the substrate surface and oxygen density has a bump near the substrate surface. These results indicate that $\mathrm{Bi}$ contained in a film precipitates as oxides near the substrate surface. As shown in Fig.2(b), the Bi peak vanishes in garnet film crystallized on a Pt underlayer with a thickness of 20nm. The FL of this film exhibits good squareness. Therefore, $B i$ precipitation at the substrate surface is one of major causes of compositional inhomogeneity in garnet film.

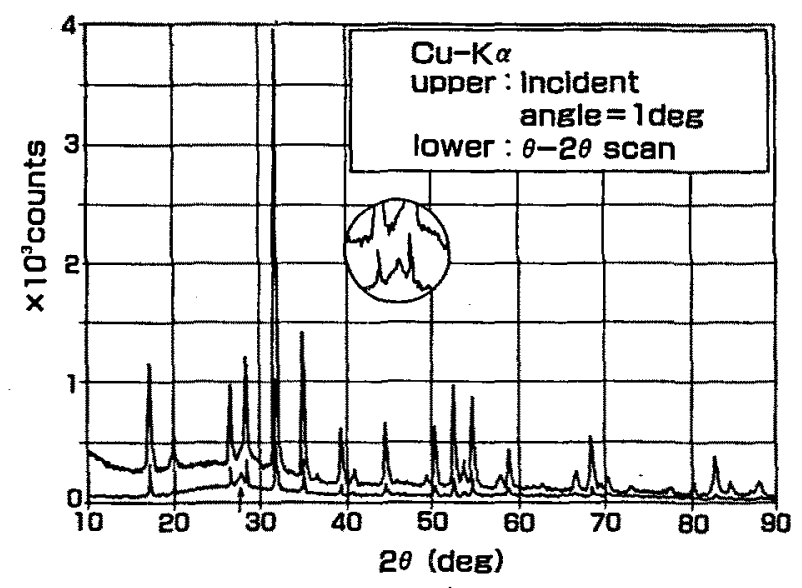

Fig. 1 XRD spectra of incident angle of 1 degree and $\theta-2 \theta$ scan. Non-garnet peak is pointed by an arrow and enlarged in a circle.
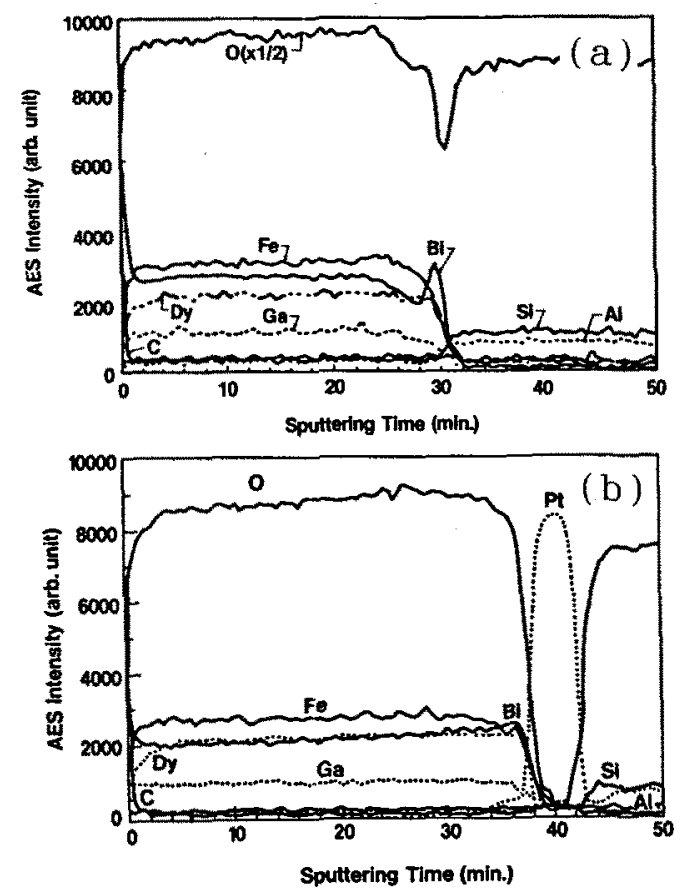

Fig.2 AES depth profiles of garnet films without((a)) and with ((b)) Pt under layer.

Are there any other inhomogeneities in garnet film? A peak in $\mathrm{Bi}$ density is found also at the left end of the vertical axis, that is, at the film surface. Although the depth of the Bi-rich region is about $1 / 30$ of the film thickness in Fig.2, this value increases to $1 / 3$ in the most extreme case. Therefore, the precipitated $\mathrm{Bi}$ at the film surface should be suppressed.

Bi had already precipitated at the film surface before annealing as shown in Fig.3(a). The AES 
signal intensity of $\mathrm{Bi}$ at the film surface is twice that which exists at the middle of the film. Though diffusion by heat of a film and/or substrate is considered to be a motive force in this phenomenon, there was no relation between a $\mathrm{Bi}$ AES intensity and substrate temperature. Sputter-etching the surface of the as-deposited film in situ under the conditions shown in Tab.1, Bi AES intensity at the film surface was reduced to $117 \%$ of that in the deep region as shown in Fig.3(b). From this result, we conclude that precipitated Bi can be effectively removed by sputter-etching. Bi precipitated again at the film surface after annealing. But a depth of $\mathrm{Bi}-\mathrm{rich}$ region in a non-etched sample decreased from $8.3 \%$ of the film thickness to $6.3 \%$ Additionally, the garnet film crystallized from the etched film was confirmed to be single phase by XRD. In one sample, we found through AFM observation denting of garnet regions, that is, voulme contraction of the film after crystallization in which amorphous regions remained[1]. So, $B i$ is thought to have precipitated again because $\mathrm{Bi}$ is squeezed out from garnet regions which are contracting. If the volume of film is contracting during deposition, the $\mathrm{Bi}$ precipitation in the

as-deposited state can be explained. Though sputter-etching after crystallization is thought to be more effective for ridding the surface of excess Bi, we feared changes of crystallinity and/or magnetic properties in the garnet film. Actually, the coercivity of $\mathrm{Bi}, \mathrm{Ga}: \mathrm{Dy} \mathrm{IG}$ film was reduced by sputter-etching after crystallization.

Next, surface morphology of the Bi,Ga:YIG film, which is used as an underlayer for Bi,Ga:DyIG film, was characterized by AFM. AFM images of $\mathrm{Bi}, \mathrm{Ga}: Y I G$ film crystallized from a non-etched and an etched film are shown in Fig.4(a) and (b), respectively.
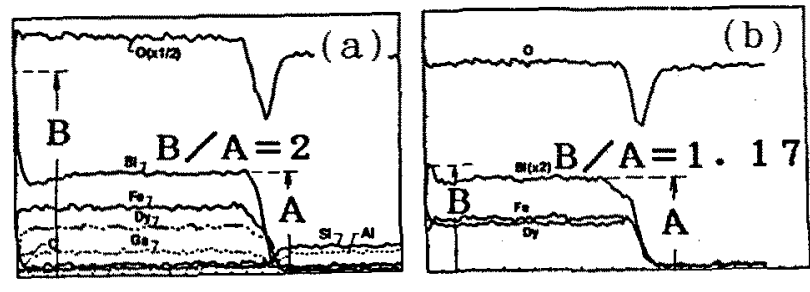

Fig. 3 AES depth profiles before annealing. (a) is the non-etched sample and (b) is sputter-etched for a period equal to $1 / 12$ of depositing time.

Obviously, top view size of roughness in Fig.4(a) is larger than that in Fig.4(b). The averaged surface roughness is $5 \mathrm{~nm}$ in Fig.4(b), which is $1 / 4$ of that in Fig.4(a). The improvement in the surface roughness is the great merit of this method. The reasons for this result are considered as follows. Because the excess $\mathrm{Bi}$ was removed from the film surface and compositional homogeneity was improved near the surface, a probability of nucleation became high and spatially homogeneous near the film surface, and the crystal growing rate also became homogeneous. As a result, the top view size of surface roughness became insignificant and the film surface became smooth. of course the oxygen vacancy densities, which influence the distribution of crack and grain boundary[2], also might be affected by etching. But we think that the rational described above is most plausible because we observed a surface roughness of $70 \%$ of a film thickness in a sample crystallized from a $f i l m$ which included $B i$ in a metal state[1].

We applied the sputter-etching method to each layer of the Bi,Ga:DyIG/Bi,Ga:YIG bi-layered film. Recorded bits on this bi-layered film were compared with those on a non-etched Bi,Ga:DyIG single layered film. As shown in Fig.5(a), bit shape and bit size are rather irregular in the garnet single layered film, and there remains regions here and there on which bits can not be recorded. Fig.5(b) shows that bits with a more regular shape and size are 

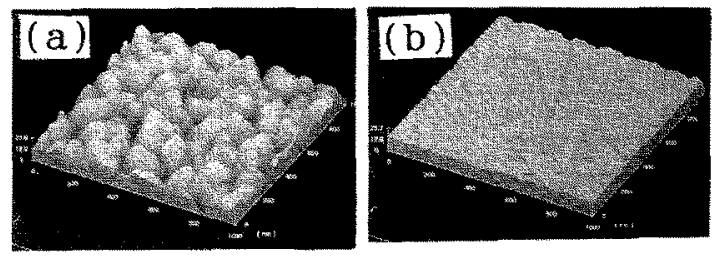

Fig.4 AFM images of non-etched ((a)) and etched((b)) Bi, Ga:YIG. Observed area is $1 \mu m x i \mu m$.

An averaged surface roughness is $20 \mathrm{~nm}$ in (a) and $5 \mathrm{~nm}$ in (b).

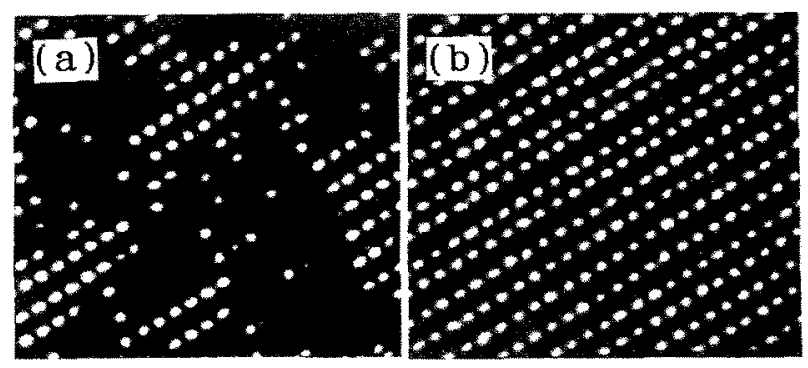

Fig.5 Recorded bits on Bi,Ga:DyIG single layered film (a)) and bi-layered garnet film( (b)). An averaged bit size is $1.5 \mu \mathrm{m}$ in (b).

recorded without dropouts on the bi-layered film. It has been reported that the garnet bi-layered structure such as $\mathrm{Bi}, \mathrm{Ga}: \mathrm{DyIG}$ on YIG[3] or Bi,Ga:DyIG on $\mathrm{Bi}, \mathrm{Ga} ; \mathrm{DyIG}[4]$ is effective in realizing a flat film surface and fine bits. A garnet bi-layered film will be deprived of these advantages if the surface of an each layer has become rough for the $B i$ precipitation at the film surface. Accordingly, the authors are convinced that the sputter-etching method presented in this report does not lose its usefulness even in a garnet bi-layered film.

\section{CONCLUSION}

The Bi-rich region observed near the surface of Bi-substituted garnet film was removed before annealing by sputter-etching for a period equal to $1 / 12$ of depositing time. The averaged surface roughness of garnet film crystallized from an etched film was $1 / 4$ of that for a non-etched sample. Bits with a regular shape and size could be recorded on a garnet bi-layered film each layer of which had been sputter-etched and crystallized.

sputter-etching the surface of the as-deposited film is a very effective method of preparing a garnet film suitable for magneto-optical recording.

\section{ACKNOWLEDGMENTS}

The authors wish to acknowledge Dr. Takizawa of Advanced Materials and Devices Research Division and Dr. Fujimoto of NHK Science and Technical Research Laboratories for motivating us to study garnet film for magneto-optical recording. The authors also acknowledge $\mathrm{Mr}$. Ishii's support in these experiments.

\section{REFERENCES}

[1]R. Sato, N. Kawamura, $T$. Tamaki, and T. Nomura:Proc. of ICF6, Tokyo,1558(1992).

[2]M. Gomi, K. Utsugi, and M. Abe: J. Magn. Soc. Jpn . , 10,173(1986) .

[3]K. Shono, T. Tamanoi, S. Kuroda, and S. Ogawa:DIGESTS of ICF6, Tokyo,428(1992).

[4]K. Shimokawa, H. Donomae, T. Mukai, N. Kawamura, T. Tamaki, R. Sato, M. Kajiura, and $T$. Nomura:Proc. of MORIS'92, J.Magn.Soc.Jpn ., 17 , Supp I., S1, 85 (1993). 